\title{
Molecular Epidemiology and Antimicrobial Susceptibility of Clostridium difficile Isolates from a University Teaching Hospital in China
}

\section{OPEN ACCESS}

Edited by:

Fatah Kashanchi,

George Mason University, USA

Reviewed by:

Jozsef Soki,

University of Szeged, Hungary

Shijian Zhang,

Dana-Farber Cancer Institute, USA

${ }^{*}$ Correspondence:

Ying-Chun Xu

xycpumch@139.com

${ }^{\dagger}$ These authors have contributed equally to this work.

Specialty section:

This article was submitted to Infectious Diseases,

a section of the journal

Frontiers in Microbiology

Received: 14 July 2016 Accepted: 28 September 2016 Published: 17 October 2016

Citation:

Cheng J-W, Xiao M, Kudinha T, Kong F, XU Z-P, Sun L-Y, Zhang L, Fan X, Xie X-L and Xu Y-C (2016)

Molecular Epidemiology and Antimicrobial Susceptibility of Clostridium difficile Isolates from

a University Teaching Hospital in China. Front. Microbiol. 7:1621. doi: 10.3389/fmicb.2016.01621

\author{
Jing-Wei Cheng ${ }^{1,2+}$, Meng Xiao ${ }^{1 \dagger}$, Timothy Kudinha ${ }^{3,4}$, Fanrong Kong $^{3}$, Zhi-Peng X ${ }^{1}$, \\ Lin-Ying Sun ${ }^{5}$, Li Zhang ${ }^{1}$, Xin Fan ${ }^{1,2}$, Xiu-Li Xie ${ }^{1}$ and Ying-Chun $\mathrm{Xu}{ }^{1 *}$
}

${ }^{1}$ Department of Clinical Laboratory, Peking Union Medical College Hospital, Chinese Academy of Medical Sciences, Beijing, China, ${ }^{2}$ Graduate School, Peking Union Medical College, Chinese Academy of Medical Sciences, Beijing, China, ${ }^{3}$ School of Biomedical Sciences, Charles Sturt University, Orange, NSW, Australia, ${ }^{4}$ Centre for Infectious Diseases and Microbiology Laboratory Services, Westmead Hospital, Sydney, NSW, Australia, ${ }^{5}$ Teaching and Research Section of Clinical Laboratory, School of Public Health, Taishan Medical School, Taian, China

While the developed world has seen a significant increase in the number of scientific articles on Clostridium difficile infection (CDI), the developing world still lags behind on this subject due to limited laboratory capacity, low awareness, and limited surveillance of this problem. As such, CDI is considered a neglected but potentially huge problem in developing countries. The major aim of this study was to systemically evaluate the utility of several molecular typing tools for $\mathrm{CDI}$, including their relevance in epidemiological studies in developing countries such as China. A total of 116 nonrepetitive toxigenic $C$. difficile isolates from Chinese patients, were studied. The isolates comprised 83 (71.6\%) A+B+CDT- isolates, 27 (23.3\%) A-B+CDT- isolates, and 6 (5.1\%) A+B+CDT+ isolates. Typing methods evaluated included multilocus variablenumber tandem-repeat analysis, PCR ribotyping, multilocus sequence typing, and sequencing of $s / p A$ and $t c d C$ genes, which identified 113, 30, 22, 18, and 8 genotypes each and exhibited discriminatory powers of 0.999, 0.916, 0.907, 0.883, and 0.765, respectively. Compared to $\mathrm{A}+\mathrm{B}+$ strains, $\mathrm{A}-\mathrm{B}+$ strains exhibited higher prevalence of drug resistance to clindamycin, erythromycin, levofloxacin, rifampicin, rifaximin, and tetracycline. Furthermore, drug resistance rates of strains with different PCR ribotypes differed, supporting the importance of molecular typing in management and control of CDI. Based on our earlier suggestion to improve the diagnostic laboratory capacity of $\mathrm{CDI}$ in developing countries, setting up efficient surveillance programs complemented by relevant molecular typing methods is warranted.

Keywords: Clostridium difficile, molecular typing, antimicrobial resistance, surveillance, China

\section{INTRODUCTION}

Clostridium difficile is a leading cause of both nosocomial and antibiotic-associated diarrhea. The clinical manifestation of C. difficile infection (CDI) ranges from asymptomatic colonization to mild diarrhea to toxic megacolon and fulminant colitis. CDI is now considered a public health threat, with a dramatic rise in the incidence and severity of CDI observed in Europe and North America in the past two decades (Rupnik et al., 2009). 
Most CDI cases have largely been attributed to the presence of the hyper-virulent $C$. difficile strain BI/NAP1/027 [restriction endonuclease analysis (REA) group BI, North American pulsefield type 1, PCR ribotype 027; McDonald et al., 2005]. Although ribotype 027 strains have caused major epidemics in North America and Europe, only sporadic cases have been reported in Asia (Collins et al., 2013). The major virulence factors for $C$. difficile are enterotoxin A (TcdA) and cytotoxin B (TcdB) (George et al., 1978). However, some strains that produce a binary toxin called $C$. difficile binary toxin $(\mathrm{CDT})$, and whose role in causing disease is not yet clear, have been described and are associated with increased virulence and recurrence rates (Popoff et al., 1988).

Knowledge of the antimicrobial susceptibility profiles and molecular types of $C$. difficile is an important first step for monitoring and understanding the epidemiology of this organism. A variety of molecular methods have been applied for genotyping of C. difficile, including PCR ribotyping, pulsed-field gel electrophoresis (PFGE), multilocus sequence typing (MLST), multilocus variable-number tandem-repeat analysis (MLVA), and sequencing of functional genes such as $s l p A$ and $t c d C$ (Collins et al., 2015). Each of these typing methods has its own merits and disadvantages, and may be applied in different occasions according to the aim and scale of the study (Killgore et al., 2008; Collins et al., 2015).

In China, there are limited studies on the molecular epidemiology and antibiotic susceptibility profiles of C. difficile, possibly due to insufficient laboratory diagnostic capacity, low awareness, and lack of high-quality surveillance systems (Collins et al., 2013; Hawkey et al., 2013; Cheng et al., 2015). However, in two previous retrospective studies, PCR ribotype 027 isolates were detected, suggesting that the threat of CDI in China is generally neglected and possibly underestimated (Cheng et al., 2016). To partly address this problem, we previously proposed a glutamate dehydrogenase-based algorithm for improving the clinical laboratory diagnostic capacity for CDI in China (Cheng et al., 2015).

The objective of this study was to evaluate the utility of different molecular typing assays for $C$. difficile, in relation to toxigenicity and antimicrobial susceptibility, using isolates obtained from one hospital in China. The usefulness of the different assays in different situations was considered, to inform decision making for improving the capacity of clinical laboratories in the management of $\mathrm{CDI}$ in developing countries like China.

\section{MATERIALS AND METHODS}

\section{Ethics}

The study was approved by the Human Research Ethics Committee of Peking Union Medical College Hospital (PUMCH) (No. PUMCHBC-C-4). Written informed consents were obtained from patients for use of the samples in research.

\section{Bacterial Isolates}

A total of 116 non-duplicate toxigenic C. difficile isolates were recovered from patients with suspected CDI in PUMCH between August 2012 and July 2015. The majority of the isolates (69.0\%; $80 / 116)$ were from the medical wards, followed by outpatient or emergency department (22.4\%; 26/116), surgical department $(6.0 \% ; 7 / 116)$, and finally intensive care units $(2.6 \% ; 3 / 116)$.

All specimens were initially tested for toxin $A / B$ using enzyme immunoassay (EIA; VIDAS C. difficile Toxin A\&B, bioMérieux, Marcy l'Etiole, France) and cultured on selective cycloserine-cefoxitin-fructose agar (CCFA) plates. Typical colonies on CCFA were identified as $C$. difficile by matrix-assisted laser desorption/ionization time-of-flight mass spectrometry (MALDI-TOF MS; Bruker Daltonics GmbH, Bremen, Germany). Only C. difficile isolates obtained from stool specimens with positive EIA results, and confirmed by MALDI-TOF MS, were included in the study.

\section{DNA Extraction, Toxin Gene Detection, and Sequencing of tcdC Gene}

DNA extraction and subsequent toxin gene detection was carried out as previously described (Cheng et al., 2015). Genotype of $t c d C$ gene was determined by comparing the obtained sequences with previous published sequences as described by Curry et al. (2007).

\section{Molecular Type Assays}

Capillary sequencer-based PCR ribotyping was performed as described by Indra et al. (2008), and ribotypes were assigned by querying the results against WEBRIBO database ${ }^{1}$. Novel ribotypes were named as "PUR" plus two Arabic numbers (e.g., PUR01).

Multilocus sequence typing was performed by sequencing seven house-keeping gene loci as previously described by Griffiths et al. (2010), and sequence type (ST) and clades of C. difficile isolates were determined by querying on official website ${ }^{2}$.

Multilocus variable-number tandem-repeat analysis was performed using the set of seven loci as proposed by van den Berg et al. (2007). Repeat numbers were analyzed using BioNumerics software v6.5 (Applied Maths, Texas, USA) for cluster analysis.

Sequence typing of the $\operatorname{sl} A$ A gene was performed as described previously by Kato et al. (2005). However, due to lack of a consistent nomenclature for $\operatorname{slp} A$ genotypes, different $\operatorname{slp} A$ genotypes and subtypes were defined as per NCBI database GenBank accession entries for $\operatorname{slp} A$ genotype descriptions (Joost et al., 2009).

\section{Antimicrobial Susceptibility Testing}

Antimicrobial susceptibility testing was performed by the agar dilution method according to Clinical and Laboratory Standards Institute (CLSI) guidelines (document M11-A8; CLSI, 2012). The following 11 antimicrobial agents were used: ciprofloxacin, clindamycin, erythromycin, levofloxacin, meropenem, metronidazole, piperacillin/tazobactam, rifampicin,

\footnotetext{
${ }^{1}$ https://webribo.ages.at/

${ }^{2}$ http://pubmlst.org/cdifficile/
} 
rifaximin, tetracycline, and vancomycin. Interpretation of testing results was based on CLSI M100-S25 (CLSI, 2015), or according to the criteria suggested by Huang et al. (2009) for the drugs whose breakpoints were not available in CLSI documents, as summarized in Supplementary Table S1. Bacteroides fragilis ATCC 25285 was used for quality control.

\section{Data Analysis}

The genetic relationships of the isolates was determined by cluster analysis using the minimum-spanning tree available in the BioNumerics software v 6.5 (Applied Maths).

To compare the discriminatory power of different molecular methods, we used an index of discriminatory power (D) based on Simpson's index of diversity (Fawley et al., 2015).

Statistical analyses were performed using SPSS software (version 17.0, IBM, New York, NY, USA). The chi-square test was applied to compare categorical variables. The level of statistical significance was defined as $P<0.05$.

\section{RESULTS}

\section{Toxigenic Types and tcdC Genotype}

Of the 116 toxigenic strains studied, 83 (71.6\%) were $t c d A$ positive, $t c d B$-positive, and $c d t A / c d t B$-negative $(A+B+C D T-)$, while $27(23.3 \%)$ were $t c d A$-negative, $t c d B$-positive, and $c d t A / c d t B$-negative $(\mathrm{A}-\mathrm{B}+\mathrm{CDT}-)$. The remaining six $(5.1 \%)$ isolates were $t c d A$-positive, $t c d B$-positive, and $c d t A / c d t B$-positive $(\mathrm{A}+\mathrm{B}+\mathrm{CDT}+$; Table $\mathbf{1})$.

Seven previously described $t c d C$ STs were identified, including tcdC-0 (40 isolates, $34.5 \%)$, tcdC-B (1 isolate, $0.86 \%), t c d C$ sc1 (2 isolates, $1.7 \%), t c d C$-sc3 (17 isolates, $14.7 \%)$, tcdC-sc7 (26 isolates, $22.4 \%$ ), $t c d C$-sc9 (26 isolates, 22.4\%), and $t c d C$ sc15 ( 1 isolate, $0.9 \%$ ). One novel tcdC genotype (3 isolates, $2.5 \%)$ was also identified, and was identical to sequence JF719680 deposited in the GenBank and named as tcdCpucl. Three genotypes, including $t c d C-s c 1, t c d C-B$, and the novel genotype $t c d C$-pucl, had an 18-bp deletion at position $330-347$, which is characteristic of ribotype 027 . In addition, the two $t c d C$-sc1 isolates had a single-nucleotide deletion at position 117.

\section{MLST}

The 116 strains were classified into 22 STs. ST54 was the most common $(n=19,16.4 \%)$, followed by ST3 ( $n=17,14.7 \%)$, ST37 ( $n=16,13.8 \%)$, ST2 and ST81 ( $n=11,9.5 \%$ each), whilst other STs were rare (prevalence $<8 \%$ ). Two novel STs, ST333 and ST334, were identified (Table 1). The majority of isolates $(n=82$, $70.1 \%$ ) belonged to clade 1 , followed by clade $4(n=28,24.1 \%)$, and only three isolates each belonged to clades 2 and 3 (Table 1).

\section{s/pA Genotypes}

By $\operatorname{slp} A$ sequencing, the 116 isolates were discriminated into 13 major genotypes and 18 subtypes (Table 1). The dominant $\underline{s l p A}$ genotype was fr-01 $(n=24,20.7 \%)$, followed by kr-03 $(n=21$, $18.1 \%)$, gr-01 ( $n=17,14.7 \%)$, yok-01 and hr-01 $(n=11,9.5 \%$ each). Two novel $\operatorname{slp} A$ subtypes, namely pus1-01 and pus2-01, were identified in the present study.

\section{Capillary Sequencer-Based PCR Ribotyping}

Thirty different PCR ribotypes were identified. The dominant ribotype was $012(n=19,16.4 \%)$, followed by ribotype 001 $(n=17,14.7 \%)$, and ribotype $017(n=16,13.8 \%$; Table 1$)$. Strains of the hyper-virulent PCR ribotype 027 ( $n=2,1.7 \%)$ were also detected. Other previously undocumented ribotypes, which we designated PUR01-PUR17, were detected. Of these novel ribotypes, ribotype PUR09 exhibited higher prevalence $(n=11$, 9.5\%) whilst the prevalence of each of the other ribotypes was low (0.9-3.4\%; Table 1).

\section{MLVA}

Using the seven loci for MLVA typing, namely A6, B7, C6, E7, F3, G8, and H9, we identified 35, 24, 37, 11, 6, 28, and 3 different alleles, amongst 116 isolates, respectively. By combination analysis of the seven loci, a total of 113 different MLVA types were identified (Figure 1D). None of the MLVA types identified comprised more than three isolates each.

\section{Relatedness of Genotypes Determined by Different Typing Methods}

Of the different typing methods used, MLVA exhibited the greatest discriminatory power, generating a $D$-value of 0.999 , followed by PCR ribotyping ( $D$-value 0.916$)$ and MLST ( $D$-value 0.907). In comparison to the above, slp $A$ and $t c d C$ sequencing exhibited lower discriminatory power $(D$-value 0.883 and 0.765 , respectively; Table 2 ).

Of note, there was a strong correlation between toxigenic types and MLST clades: all 82 MLST clade 1 isolates were $\mathrm{A}+\mathrm{B}+\mathrm{CDT}-$. Among 28 MLST clade 4 isolates, 27 (96.4\%) were of toxigenic type $\mathrm{A}-\mathrm{B}+\mathrm{CDT}-$ and only one isolate $(3.6 \%)$ was $\mathrm{A}+\mathrm{B}+\mathrm{CDT}-$. Furthermore, all $\mathrm{A}+\mathrm{B}+\mathrm{CDT}+$ isolates were only found in MLST clades 2 and 3 (Table $\mathbf{1}$ ).

There was also some association between $t c d C$ genotyping results and MLST clades: $t c d C$-WT $(39 / 82,47.6 \%)$ and $t c d C$-sc9 $(25 / 82 ; 30.5 \%)$ isolates were the commonest $t c d C$ genotype in MLST clade 1, and were rarely represented (one isolate each) in other MLST clades. All the $17 \mathrm{~A}+\mathrm{B}+\mathrm{CDT}-$ isolates which belonged to the second commonest ST in MLST clade 1, i.e., ST3 (17/82, 20.7\%), were $t c d C$-sc3. In addition, 96.3\% (26/27) $\mathrm{A}-\mathrm{B}+\mathrm{CDT}-$ isolates were $t c d C$-sc7 (Table 1; Figure 1A).

Of 18 different $\operatorname{slp} A$ genotypes/subtypes identified, only two were shared by different MLST clades, namely hr-01 shared by MLST clade $1(n=10)$ and clade $4(n=1)$, and $\mathrm{kr}-04$ shared by MLST clade $1(n=1)$ and clade $3(n=1)$. Other $s l p A$ genotypes, although shared by different MLST STs, were unique amongst MLST clades (Table 1; Figure 1B).

PCR ribotyping exhibited higher discriminatory power than MLST, and none of the PCR ribotypes were shared by different MLST clades (Figure 1C). Four ribotypes were identified amongst six $\mathrm{A}+\mathrm{B}+\mathrm{CDT}+$ isolates. The two ribotype type 027 isolates, which have been previously reported by our group 
TABLE 1 | Multilocus sequence typing (MLST), ribotype, slpA, tcdC, and toxin genotypes of the 116 Clostridium difficile clinical isolates.

\begin{tabular}{|c|c|c|c|c|c|c|}
\hline MLST clade & MLST ST & Ribotype & s/pA genotype & tcdC genotype & Toxin gene & No. of isolates \\
\hline \multirow[t]{29}{*}{ Clade 1} & ST2 & 14 & hr-01 & $\operatorname{sc} 9$ & $\mathrm{~A}+\mathrm{B}+\mathrm{CDT}-$ & 2 \\
\hline & & & & WT & $\mathrm{A}+\mathrm{B}+\mathrm{CDT}-$ & 4 \\
\hline & & & pus1-01 & WT & $\mathrm{A}+\mathrm{B}+\mathrm{CDT}-$ & 1 \\
\hline & & 20 & hr-01 & $\operatorname{sc} 9$ & $\mathrm{~A}+\mathrm{B}+\mathrm{CDT}-$ & 1 \\
\hline & & & & $\mathrm{WT}$ & $\mathrm{A}+\mathrm{B}+\mathrm{CDT}-$ & 1 \\
\hline & & PUR02 & hr-01 & $\operatorname{sc} 9$ & $\mathrm{~A}+\mathrm{B}+\mathrm{CDT}-$ & 1 \\
\hline & & PUR03 & cc12078-01 & $\mathrm{WT}$ & $\mathrm{A}+\mathrm{B}+\mathrm{CDT}-$ & 1 \\
\hline & ST3 & 1 & gr-01 & $\mathrm{sc} 3$ & $\mathrm{~A}+\mathrm{B}+\mathrm{CDT}-$ & 17 \\
\hline & ST8 & 2 & yok-01 & $\mathrm{WT}$ & $\mathrm{A}+\mathrm{B}+\mathrm{CDT}-$ & 3 \\
\hline & & PUR04 & yok-01 & WT & $\mathrm{A}+\mathrm{B}+\mathrm{CDT}-$ & 1 \\
\hline & & PUR05 & yok-01 & $\mathrm{WT}$ & $\mathrm{A}+\mathrm{B}+\mathrm{CDT}-$ & 4 \\
\hline & ST27 & 39 & sh-01 & WT & $\mathrm{A}+\mathrm{B}+\mathrm{CDT}-$ & 1 \\
\hline & ST35 & 46 & og39-01 & WT & $\mathrm{A}+\mathrm{B}+\mathrm{CDT}-$ & 8 \\
\hline & ST42 & 106 & $\mathrm{hr}-02$ & WT & $\mathrm{A}+\mathrm{B}+\mathrm{CDT}-$ & 5 \\
\hline & ST51 & PUR07 & yok-01 & $\mathrm{B}^{\mathrm{a}}$ & $\mathrm{A}+\mathrm{B}+\mathrm{CDT}-$ & 1 \\
\hline & ST54 & 12 & $\mathrm{kr}-03$ & $\operatorname{sc} 9$ & $\mathrm{~A}+\mathrm{B}+\mathrm{CDT}-$ & 16 \\
\hline & & & & WT & $\mathrm{A}+\mathrm{B}+\mathrm{CDT}-$ & 3 \\
\hline & ST55 & 70 & $\mathrm{kr}-04$ & $\operatorname{sc} 9$ & $\mathrm{~A}+\mathrm{B}+\mathrm{CDT}-$ & 1 \\
\hline & & PUR08 & $\mathrm{kr}-03$ & $\operatorname{sc} 9$ & $\mathrm{~A}+\mathrm{B}+\mathrm{CDT}-$ & 1 \\
\hline & ST91 & PUR10 & ar-01 & WT & $\mathrm{A}+\mathrm{B}+\mathrm{CDT}-$ & 1 \\
\hline & ST98 & PUR11 & pus1-01 & WT & $\mathrm{A}+\mathrm{B}+\mathrm{CDT}-$ & 1 \\
\hline & ST129 & PUR13 & $\mathrm{xr}-03$ & $\operatorname{sc} 9$ & $\mathrm{~A}+\mathrm{B}+\mathrm{CDT}-$ & 1 \\
\hline & & & & WT & $\mathrm{A}+\mathrm{B}+\mathrm{CDT}-$ & 1 \\
\hline & ST233 & PUR14 & yok-01 & $\operatorname{sc} 9$ & $\mathrm{~A}+\mathrm{B}+\mathrm{CDT}-$ & 1 \\
\hline & ST278 & PUR15 & $\mathrm{kr}-03$ & WT & $\mathrm{A}+\mathrm{B}+\mathrm{CDT}-$ & 1 \\
\hline & ST286 & PUR16 & pus2-01 & $\mathrm{WT}$ & $\mathrm{A}+\mathrm{B}+\mathrm{CDT}-$ & 1 \\
\hline & ST289 & PUR17 & hr-01 & $\operatorname{sc} 9$ & $\mathrm{~A}+\mathrm{B}+\mathrm{CDT}-$ & 1 \\
\hline & ST333 & 2 & yok-01 & WT & $\mathrm{A}+\mathrm{B}+\mathrm{CDT}-$ & 1 \\
\hline & ST334 & PUR12 & gc11-01 & WT & $\mathrm{A}+\mathrm{B}+\mathrm{CDT}-$ & 1 \\
\hline \multirow[t]{2}{*}{ Clade 2} & ST1 & 27 & gc8-03 & $\mathrm{sc} 1^{\mathrm{a}}$ & $\mathrm{A}+\mathrm{B}+\mathrm{CDT}+$ & 2 \\
\hline & & PUR01 & gc8-03 & WT & $\mathrm{A}+\mathrm{B}+\mathrm{CDT}+$ & 1 \\
\hline \multirow[t]{2}{*}{ Clade 3} & ST5 & 23 & $\mathrm{kr}-04$ & $\operatorname{puc}^{a}$ & $\mathrm{~A}+\mathrm{B}+\mathrm{CDT}+$ & 1 \\
\hline & & 63 & j52-01 & puct $^{a}$ & $\mathrm{~A}+\mathrm{B}+\mathrm{CDT}+$ & 2 \\
\hline \multirow[t]{5}{*}{ Clade 4} & ST37 & 17 & $\mathrm{fr}-01$ & $\mathrm{sc} 7$ & $\mathrm{~A}-\mathrm{B}+\mathrm{CDT}-$ & 12 \\
\hline & & & & $\operatorname{sc} 9$ & $\mathrm{~A}-\mathrm{B}+\mathrm{CDT}-$ & 1 \\
\hline & & & $\mathrm{fr}-06$ & $\mathrm{sc7}$ & $\mathrm{A}-\mathrm{B}+\mathrm{CDT}-$ & 3 \\
\hline & ST81 & PUR09 & $\mathrm{fr}-01$ & $\mathrm{sc} 7$ & $\mathrm{~A}-\mathrm{B}+\mathrm{CDT}-$ & 11 \\
\hline & ST332 & PUR06 & hr-01 & sc15 & $\mathrm{A}+\mathrm{B}+\mathrm{CDT}-$ & 1 \\
\hline
\end{tabular}

atcdC-sc1, tcdC-B, and tcdC-puc1 had 18-bp deletion at position 330-347; tcdC-sc1 had a single-nucleotide deletion at position 117.

(Cheng et al., 2016), belonged to MLST ST1 (clade 2), slpA genotype gc8-03, and $t c d C$ genotype $t c d C$-sc1 (having 18-bp deletion at position $330-347$, and single-nucleotide deletion at position 117 versus wild-type; Table 1). However, the PUR01 $(n=1) \mathrm{A}+\mathrm{B}+\mathrm{CDT}+$ isolate, which although belonged to MLST ST1 and was of $\operatorname{slp} A$ genotype gc8-03, carried the wild-type $t c d C$ gene (Table 1). In contrast, ribotype $63(n=2)$ and $23(n=1)$ $\mathrm{A}+\mathrm{B}+\mathrm{CDT}+$ isolates belonged to MLST ST5 (clade 3 ) and $t c d C$ genotype $t c d C$-puc1 (having 18-bp deletion at position 330-347, but with no single-nucleotide deletion at position 117), but were slpA genotype j52-01 and kr-04, respectively (Table 1). There were only two ribotypes identified in $27 \mathrm{~A}-\mathrm{B}+\mathrm{CDT}-$ isolates, namely ribotype $17(n=16,59.3 \%)$ and ribotype PUR09 $(n=11$,
40.7\%; Table 1). Ribotypes identified in $\mathrm{A}+\mathrm{B}+\mathrm{CDT}-$ isolates were much more diverse (24 ribotypes in 83 isolates), of which ribotype $012(n=19,22.9 \%)$ and $001(n=17,20.5 \%)$ were the commonest (Table 1).

Multilocus variable-number tandem-repeat analysis exhibited the highest discriminatory power amongst all typing methods used (Figure 1D). The minimum spanning tree generated by MLVA also indicated a general phylogenetic relatedness of MLVA typing results and MLST clades (Figure 1D). As can be seen clearly in Figure 1D, using the MLVA spanning tree, MLST clade 1 was separated into two major branches by MLST clade 4. However, discrepancies between MLVA and MLST were also observed. For example, by MLVA analysis, the three MLST 


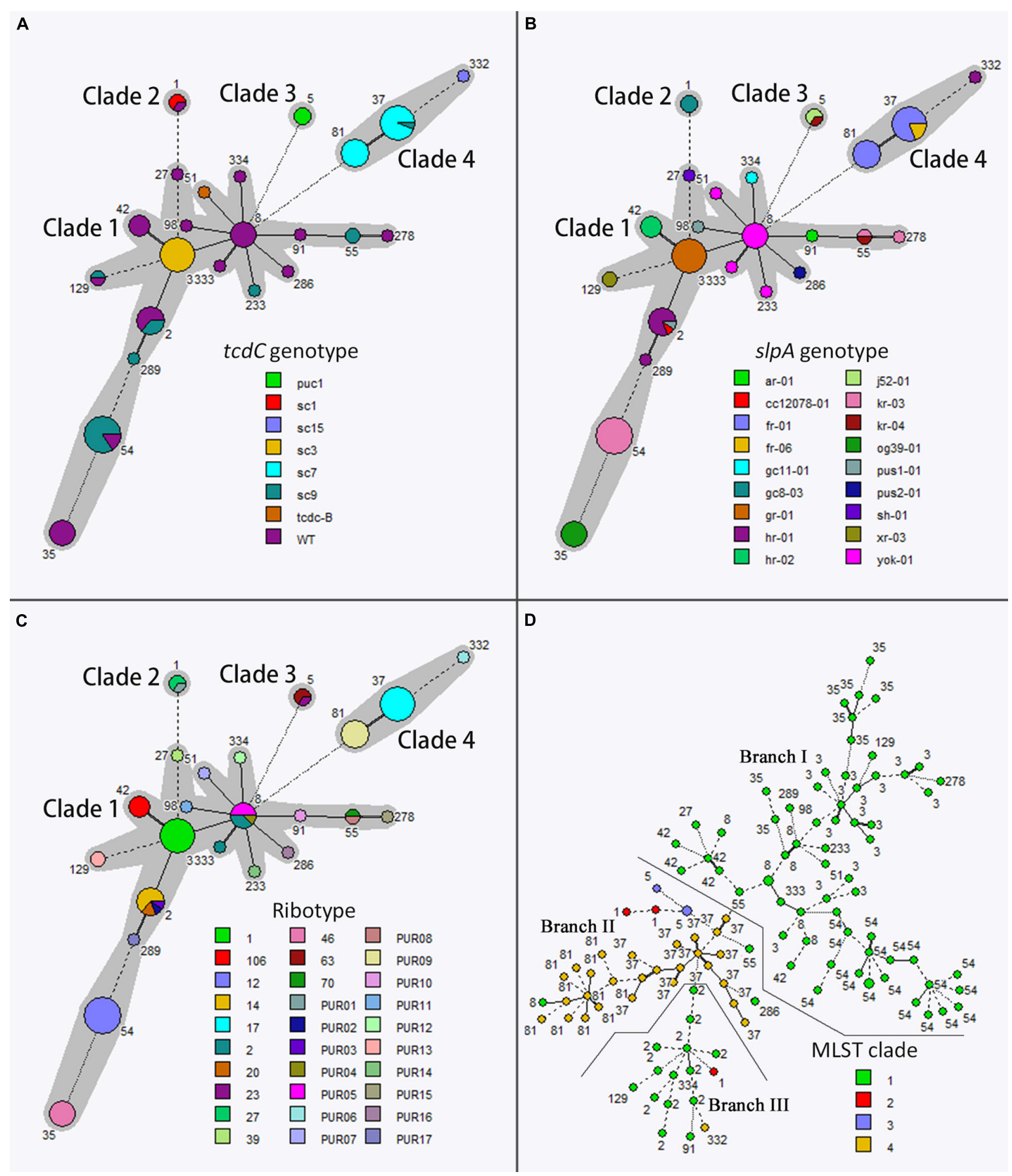

FIGURE 1 | Minimum spanning tree analysis based on allelic profiles of multilocus sequence typing (MLST) (A,B,C) and Multilocus variable-number tandem-repeat analysis (MLVA) (D) data. Each circle in (A,B,C) corresponds to a MLST ST, and each circle in (D) corresponds to a MLVA genotype. Different circle colors represent tcdC genotype, s/pA genotype, PCR ribotype, and MLST clade in (A,B,C,D), respectively. The gray halo surrounding the MLST STs in (A-C) denotes STs belonging to different MLST clades. The lines between circles indicate the similarity between profiles: bold line, six of seven MLST alleles/MLVA loci in common; normal line, five alleles/loci in common; dashed line, four alleles/loci in common; dotted line, $\leq 3$ alleles/loci.

ST1 isolates (red circles in Figure 1D) were classified into two different branches: two isolates were more phylogenetically related to MLST clade 3, but one isolate was more closely related to MLST ST2 of MLST clade 1 (Figure 1D).

\section{Antimicrobial Susceptibilities}

The minimum inhibitory concentrations (MICs) of 11 antimicrobial agents for the 116 isolates are summarized in Table 3. All the isolates were susceptible to metronidazole, 
TABLE 2 | Discriminatory power of the five typing methods used in this study.

\begin{tabular}{lccc}
\hline Method & $\begin{array}{c}\text { No. of } \\
\text { genotypes }\end{array}$ & $\begin{array}{c}\text { Index of } \\
\text { discrimination }\end{array}$ & $\begin{array}{c}\text { 95\% confidence } \\
\text { interval }\end{array}$ \\
\hline MLVA & 113 & 0.999 & $0.999-1$ \\
Ribotyping & 30 & 0.916 & $0.895-0.938$ \\
MLST & 22 & 0.907 & $0.888-0.926$ \\
slpA genotyping & 18 & 0.883 & $0.858-0.908$ \\
$t c d C$ genotyping & 8 & 0.765 & $0.731-0.798$ \\
\hline
\end{tabular}

vancomycin, meropenem, piperacillin/tazobactam, but resistant to ciprofloxacin. Co-resistance to erythromycin, clindamycin, and levofloxacin was observed in $41.3 \%$ of the isolates. High-level resistance to erythromycin (MIC $>128 \mathrm{mg} / \mathrm{L}$ ) and clindamycin $(\mathrm{MIC}>128 \mathrm{mg} / \mathrm{L})$ was detected in $63.8 \%$ and $55.2 \%$ of the 116 isolates, respectively. The MICs of rifampin were consistent with those of rifaximin, and were either $\leq 0.064 \mathrm{mg} / \mathrm{L}$ or $>256 \mathrm{mg} / \mathrm{L}$. Moreover, $25.9 \%$ of the isolates were resistant to tetracycline.

Drug resistance rates for $\mathrm{A}-\mathrm{B}+$ isolates for the antibiotics levofloxacin, rifampicin, rifaximin, and tetracycline, were significantly higher than that of $\mathrm{A}+\mathrm{B}+$ isolates $(P<0.001$; Table 3). In addition, varied antimicrobial resistance rates were observed amongst different molecular types. For instance, ribotypes 012, 017, and PUR09 isolates exhibited higher resistance rates to clindamycin and erythromycin compared to ribotype 001 and other ribotype isolates (Figure 2). Moreover, ribotype 017 and PUR09 isolates showed higher prevalence rate of resistance to levofloxacin and tetracycline, compared to other ribotype isolates. Ribotype 017 isolates also had higher rate of resistance to rifampicin and rifaximin (Figure 2).

\section{DISCUSSION}

Clostridium difficile infection has become a significant public health threat in the developed world, with substantial increase in morbidity and mortality reported since the early 2000s. However, due to inadequate laboratory diagnostic capacity, little is known about the magnitude of the CDI problem in developing countries like China, and hence the problem may be much underestimated (Collins et al., 2013; Hawkey et al., 2013; Cheng et al., 2016). In this regard, our group has previously attempted to improve the diagnosis of CDI in China by proposing a practical workflow for future CDI laboratory diagnosis (Cheng et al., 2015). Furthermore, the lack of high-quality CDI surveillance systems and epidemiology studies in developing countries contributes to the low awareness of CDI. Thus further studies are needed to address this problem.

Molecular typing methods are essential for understanding the epidemiology of $C$. difficile, and are especially important as part of an active surveillance and hospital infection control strategy for this organism. To date, several typing methods have been used to study the epidemiology of $C$. difficile, and there are pros and cons for each method. Most studies evaluating the utility of different molecular typing methods for CDI are from the developed world. In the present study, we evaluated the performance and utility

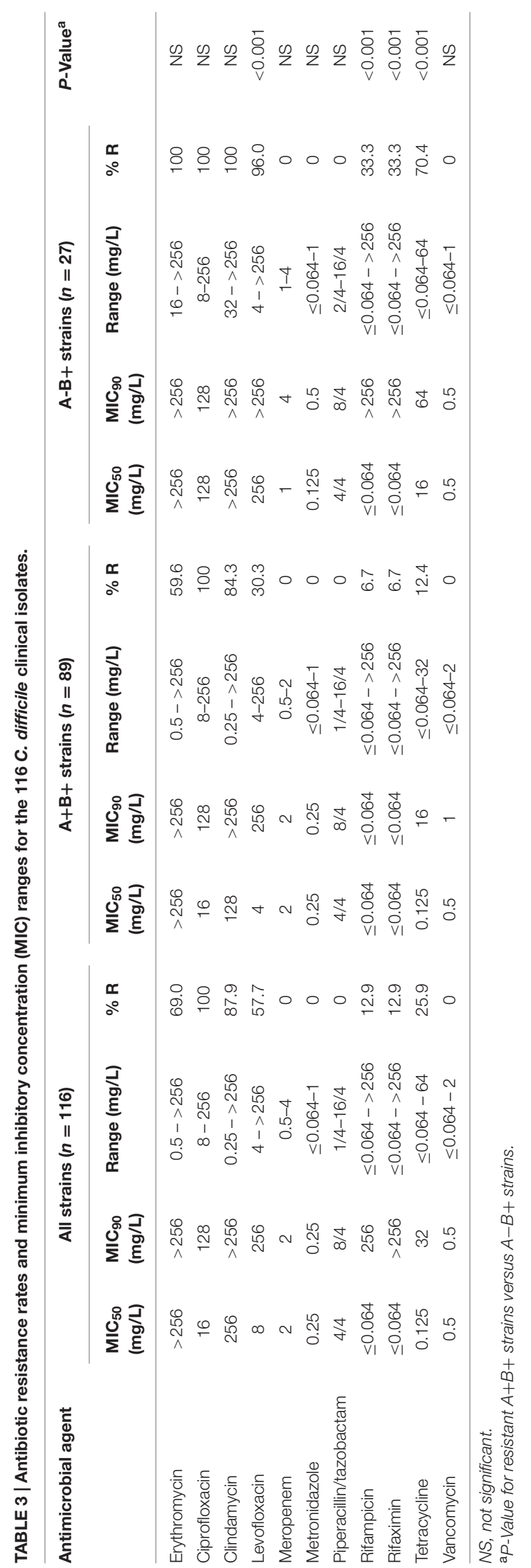




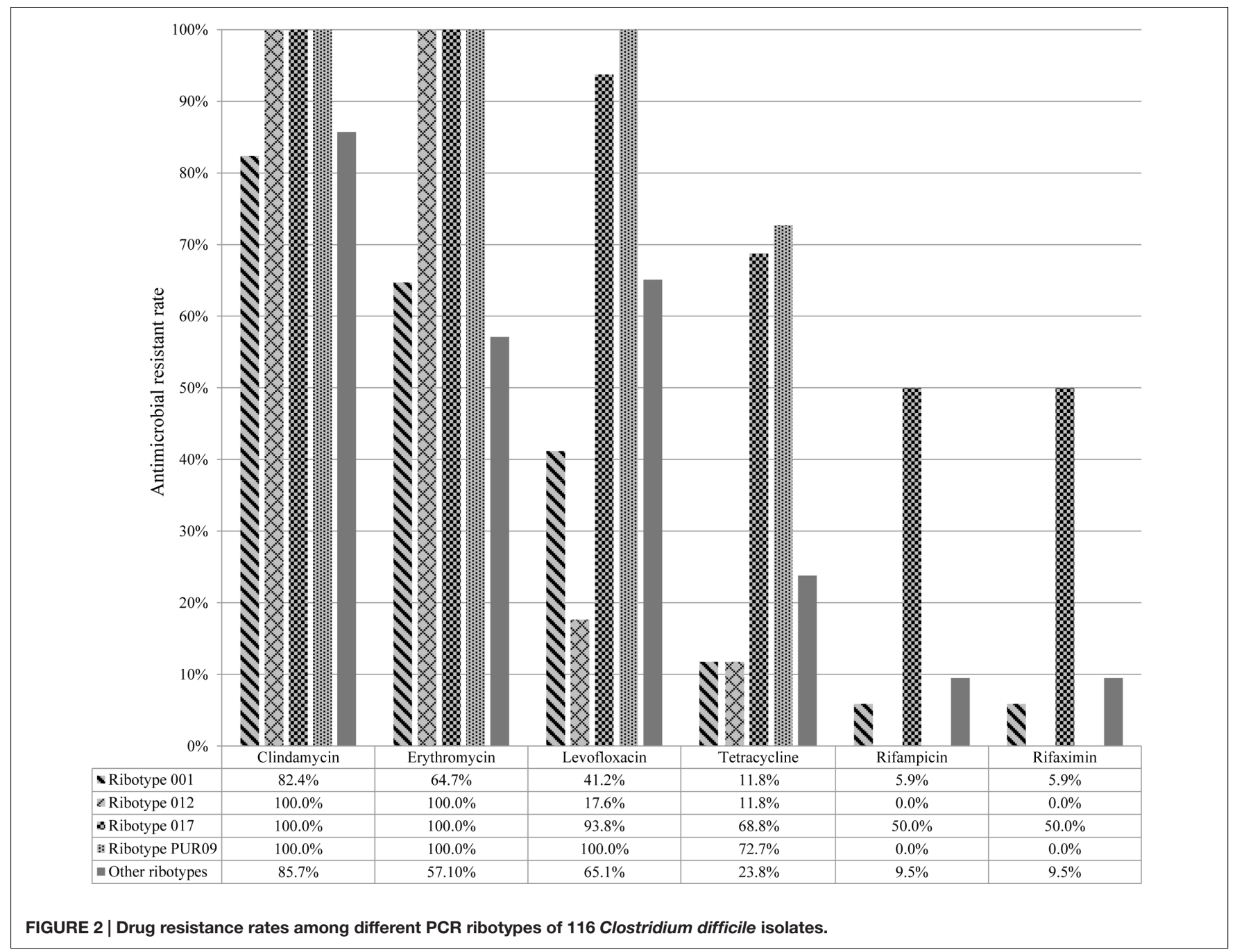

of the most commonly used genotypic assays for C. difficile, including toxigenic typing, $t c d C$ genotyping, $s l p A$ genotyping, MLST, PCR-ribotyping, and MLVA.

Although the dominant $C$. difficile toxigenic type is $\mathrm{A}+\mathrm{B}+$, there has been an increase in the prevalence and significance of the A-B+ type in some regions of the world (Kim et al., 2008; King et al., 2015). In this study, $23.3 \%$ of the isolates were $\mathrm{A}-\mathrm{B}+\mathrm{CDT}-$, which is in agreement with previous studies in China in which the average prevalence rate of $\mathrm{A}-\mathrm{B}+$ strains is $21.4 \%$ (201/937), ranging from 0 to $53.6 \%$ by geographic distribution (Huang et al., 2009, 2010; Dong et al., 2013; Hawkey et al., 2013; Yan et al., 2013; Chen et al., 2014; Fang et al., 2014; Wang et al., 2014; Zhou et al., 2014; Cheng et al., 2015; Ye et al., 2015; Tian et al., 2016). Interestingly, infection with CDTpositive $C$. difficile strains has been reported to be associated with higher mortality and recurrence rates (Stewart et al., 2013). In this study, $5.1 \%(6 / 116)$ isolates were $A+B+C D T+$, which is slightly higher than in previous studies in Shanghai $(1.6 \%$, 2/110) and Zhejiang provinces (0\%, 0/82; Huang et al., 2010; Fang et al., 2014). However, the overall prevalence of $\mathrm{A}+\mathrm{B}+\mathrm{CDT}+$ C. difficile strains in China is much lower than in North America and Europe (Bassetti et al., 2012), possibly due to low lab diagnostic capacity and subsequent lack of awareness amongst medical personnel. By $t c d C$ sequencing, five of six $\mathrm{A}+\mathrm{B}+\mathrm{CDT}+$ isolates detected in this study had an 18-bp deletion at nucleotides $330-347$, but only two ribotype 027 isolates, characterized by a single deletion at nucleotide 117 , were detected.

The predominant ribotype and ST identified in the present study was ribotype $012 / \mathrm{ST} 54(16.4 \%)$, followed by ribotypes 001/ST3 (14.7\%) and 017/ST37 (13.8\%), whilst the commonest ribotype is ribotype 012 reported overall in China (100 of 589 isolates, 17.0\%), and ST54, ST3, ST35, and ST37 (prevalence of $21.4,12.6,11.0$, and 9.4 of 682 isolates tested, respectively) rank the top four STs (Huang et al., 2009, 2010; Dong et al., 2013; Hawkey et al., 2013; Yan et al., 2013; Chen et al., 2014; Fang et al., 2014; Wang et al., 2014; Zhou et al., 2014; Cheng et al., 2015; Ye et al., 2015; Tian et al., 2016). The remaining ribotypes/STs were distributed sporadically among different departments and with a lower prevalence. Generally, there was no obvious correlation between clonal clusters of the organism generated from the different departments during the study period, suggesting that a wide variety of strains are implicated in this hospital. However, 
TABLE 4 | Comparison and suggested usage of typing methods employed in the present study.

\begin{tabular}{|c|c|c|c|c|c|}
\hline Typing method & Reproducibility & $\begin{array}{l}\text { Inter- } \\
\text { laboratory } \\
\text { exchange }\end{array}$ & $\begin{array}{l}\text { Discriminatory } \\
\text { power }\end{array}$ & $\begin{array}{l}\text { Running } \\
\text { costs }\end{array}$ & Suggested usage \\
\hline Toxigenic typing & +++ & ++ & + & + & Toxin status confirmation and risk assessment for virulence \\
\hline tcdC genotyping & +++ & + & ++ & + & Hypervirulence strain detection \\
\hline s/pA genotyping & +++ & + & ++ & + & Regional epidemiology for vaccine development \\
\hline MLST & +++ & +++ & ++ & +++ & Epidemiology surveillance (high costs); phylogenetic studies \\
\hline Ribotyping & +++ & + & ++ & + & $\begin{array}{l}\text { Epidemiology surveillance (low costs); infection prevention and } \\
\text { control }\end{array}$ \\
\hline MLVA & +++ & ++ & +++ & ++ & Detection of local outbreaks \\
\hline
\end{tabular}

due to lack of consistent data and limited lab diagnostic capacity for CDI at the hospital concerned, these findings may be biased.

Although the major molecular types identified in different C. difficile studies were similar, the prevalence of different molecular types varied amongst studies in China. In agreement to our findings, ribotype $017 \mathrm{C}$. difficile strains have been reported in several Asian countries (Collins et al., 2013). However, in North America and Europe, many CDI cases have been attributed to $C$. difficile ribotype 027 and 078 strains which have been described in many hospitals, and were particularly associated with outbreaks or epidemics (Bassetti et al., 2012). The major clone implicated in epidemics varies according to geographic locale, highlighting the significance of establishing a local surveillance network for CDI.

The emergence of increased antibiotic resistance in different C. difficile strain types worldwide may be a contributing factor to increased occurrence of CDI outbreaks in several hospitals. Although several studies have reported on the increasing MIC for metronidazole and vancomycin in C. difficile (Baines et al., 2008; Adler et al., 2015), both drugs showed high in vitro activity against all the isolates in our study. Furthermore, all strains were also susceptible to meropenem and piperacillin/tazobactam. Although a small number (12.9\%) of C. difficile strains with highlevel resistance to rifampin and rifaximin was observed in the present study, the rate was lower than the 25-29\% reported in Shanghai, China (Huang et al., 2009, 2010).

The high rate of drug resistance for ciprofloxacin (100\%), erythromycin (69\%), and clindamycin (87.9\%) among the studied isolates suggests that use of these antibiotics may be a risk factor for the development of CDI in this geographic locale (Bassetti et al., 2012). In addition, $A-B+$ strains in the present study exhibited higher drug resistance rates compared to $\mathrm{A}+\mathrm{B}+$ strains for the antibiotics clindamycin, erythromycin, levofloxacin, rifampicin, rifaximin, and tetracycline. Thus toxigenic typing may be valuable for understanding antibiotic resistance in the management and control of CDI. Furthermore, the drug resistance rates of strains of different PCR ribotypes differed. These findings emphasize the importance of implementing active surveillance and molecular epidemiologic studies on CDI.

All the typing methods used in this study have the advantage of high reproducibility (Table 4). MLVA exhibited extremely high discriminatory power, and thus may be a potential valuable tool for investigating CDI outbreaks (Eyre et al., 2013b). However, a reasonable cut-off value is needed since $C$. difficile isolates from the same outbreak may belong to genetically closely related but different MLVA types (Table 4) (Marsh et al., 2006).

Based on our findings, we recommend PCR ribotyping as the method of choice for epidemiological surveillance and infection prevention and control, as it delivers high discrimination, accuracy, and reproducibility. However, more work is needed for its standardization and database construction (Table 4) (Indra et al., 2008; Fawley et al., 2015). Although MLST also offers high discriminatory power index and easy inter-laboratory data comparison which may be useful for epidemiology surveillance and phylogenetic studies, the high cost is prohibitive (Table 4) (Killgore et al., 2008; Collins et al., 2015; Tian et al., 2016).

Sequencing of the $\operatorname{slp} A$ gene is valuable for comparison of C. difficile strains implicated in epidemics in diverse areas because the typing results are reproducible and can easily be shared. In addition, as the $\operatorname{slp} A$ gene is related to the strain's serogroup, this typing method could be useful in vaccine development (Kato et al., 2005; Tian et al., 2016). tcdC genotyping had the lowest $D$-value, but sequencing of this gene may be used as an indicator for the hyper-virulent $C$. difficile clones, e.g., PCR ribotype 027/078 isolates (Curry et al., 2007).

Several other $C$. difficile typing methods apart from the ones applied in this study have been used, including PFGE, REA, and PCR-restriction fragment length polymorphism (RFLP). Unfortunately, these restriction enzyme-agarose gel electrophoresis-based methods have poor reproducibility, and are difficult to standardize for result interpretation and inter-laboratory data exchange (Killgore et al., 2008; Collins et al., 2015). Recent advances in whole-genome sequencing of $C$. difficile has the potential to provide even greater epidemiological information, but is still premature for use in large scale epidemiologic studies (Eyre et al., 2013a; Knight et al., 2015).

\section{CONCLUSION}

Our study is the most systematic study to integrate molecular epidemiology and antibiotic susceptibility testing, and provides comprehensive data for a better understanding of CDI in China. The findings highlight the importance of active surveillance using molecular typing techniques for better management and control of CDI in developing countries. Different molecular typing assays could be used depending on the scale and aim of the surveillance. 


\section{AUTHOR CONTRIBUTIONS}

J-WC, MX, TK, and FK wrote the manuscript; Z-PX, $\mathrm{LZ}$, and XF collaborated in molecular investigations of the strains; L-YS and X-LX summarized the patient's medical records; Y-CX designed and supervised the study.

\section{FUNDING}

This study was financially supported by a Natural Science Foundation of China (grant number 81501807), a Beijing Outstanding Talents Cultivation Program (2015000020124G071), and a National Research Special Fund for Public

\section{REFERENCES}

Adler, A., Miller-Roll, T., Bradenstein, R., Block, C., Mendelson, B., Parizade, M., et al. (2015). A national survey of the molecular epidemiology of Clostridium difficile in Israel: the dissemination of the ribotype 027 strain with reduced susceptibility to vancomycin and metronidazole. Diagn. Microbiol. Infect. Dis. 83, 21-24. doi: 10.1016/j.diagmicrobio.2015.05.015

Baines, S. D., O'connor, R., Freeman, J., Fawley, W. N., Harmanus, C., Mastrantonio, P., et al. (2008). Emergence of reduced susceptibility to metronidazole in Clostridium difficile. J. Antimicrob. Chemother. 62, 1046-1052. doi: $10.1093 / \mathrm{jac} / \mathrm{dkn} 313$

Bassetti, M., Villa, G., Pecori, D., Arzese, A., and Wilcox, M. (2012). Epidemiology, diagnosis and treatment of Clostridium difficile infection. Expert Rev. Anti. Infect. Ther. 10, 1405-1423. doi: 10.1586/eri.12.135

Chen, Y. B., Gu, S. L., Wei, Z. Q., Shen, P., Kong, H. S., Yang, Q., et al. (2014). Molecular epidemiology of Clostridium difficile in a tertiary hospital of China. J. Med. Microbiol. 63, 562-569. doi: 10.1099/jmm.0. 068668-0

Cheng, J. W., Xiao, M., Kudinha, T., Xu, Z. P., Hou, X., Sun, L. Y., et al. (2016). The first two Clostridium difficile ribotype 027/ST1 isolates identified in Beijing, China-an emerging problem or a neglected threat? Sci. Rep. 6:18834. doi: 10.1038/srep 18834

Cheng, J. W., Xiao, M., Kudinha, T., Xu, Z. P., Sun, L. Y., Hou, X., et al. (2015). The role of Glutamate Dehydrogenase (GDH) testing assay in the diagnosis of Clostridium difficile infections: a high sensitive screening test and an essential step in the proposed laboratory diagnosis workflow for developing countries like China. PLoS ONE 10:e0144604. doi: 10.1371/journal.pone. 0144604

CLSI (2012). Methods for Antimicrobial Susceptibility Testing of Anaerobic Bacteria, M11-A8, 8th Edn. Wayne, PA: Clinical and Laboratory Standards Institute.

CLSI (2015). Performance Standards for Antimicrobial Susceptibility Testing; Twenty-Fifth Informational Supplement, M100-S25. Wayne, PA: Clinical and Laboratory Standards Institute.

Collins, D. A., Elliott, B., and Riley, T. V. (2015). Molecular methods for detecting and typing of Clostridium difficile. Pathology 47, 211-218. doi: 10.1097/PAT.0000000000000238

Collins, D. A., Hawkey, P. M., and Riley, T. V. (2013). Epidemiology of Clostridium difficile infection in Asia. Antimicrob. Resist. Infect. Control 2:21. doi: 10.1186/2047-2994-2-21

Curry, S. R., Marsh, J. W., Muto, C. A., O’leary, M. M., Pasculle, A. W., and Harrison, L. H. (2007). tcdC genotypes associated with severe TcdC truncation in an epidemic clone and other strains of Clostridium difficile. J. Clin. Microbiol. 45, 215-221. doi: 10.1128/JCM.01599-06

Dong, D., Zhang, L., Chen, X., Jiang, C., Yu, B., Wang, X., et al. (2013). Antimicrobial susceptibility and resistance mechanisms of clinical Clostridium difficile from a Chinese tertiary hospital. Int. J. Antimicrob. Agents 41, 80-84. doi: 10.1016/j.ijantimicag.2012.08.011
Welfare Industry of Health of China (grant number 201402001).

\section{ACKNOWLEDGMENTS}

This work was presented in part at the 26th European Congress of Clinical Microbiology and Infectious Diseases (ECCMID), Amsterdam, Netherlands, April 2016, Abstract eP0136.

\section{SUPPLEMENTARY MATERIAL}

The Supplementary Material for this article can be found online at: http://journal.frontiersin.org/article/10.3389/fmicb. 2016.01621

Eyre, D. W., Cule, M. L., Wilson, D. J., Griffiths, D., Vaughan, A., O'connor, L., et al. (2013a). Diverse sources of $C$. difficile infection identified on whole-genome sequencing. N. Engl. J. Med. 369, 1195-1205. doi: 10.1056/NEJMoa1216064

Eyre, D. W., Fawley, W. N., Best, E. L., Griffiths, D., Stoesser, N. E., Crook, D. W., et al. (2013b). Comparison of multilocus variable-number tandem-repeat analysis and whole-genome sequencing for investigation of Clostridium difficile transmission. J. Clin. Microbiol. 51, 4141-4149. doi: 10.1128/JCM.01095-13

Fang, W. J., Jing, D. Z., Luo, Y., Fu, C. Y., Zhao, P., Qian, J., et al. (2014). Clostridium difficile carriage in hospitalized cancer patients: a prospective investigation in eastern China. BMC Infect. Dis. 14:523. doi: 10.1186/1471-2334-14-523

Fawley, W. N., Knetsch, C. W., Maccannell, D. R., Harmanus, C., Du, T., Mulvey, M. R., et al. (2015). Development and validation of an internationallystandardized, high-resolution capillary gel-based electrophoresis PCR-ribotyping protocol for Clostridium difficile. PLoS ONE 10:e0118150. doi: 10.1371/journal.pone.0118150

George, R. H., Symonds, J. M., Dimock, F., Brown, J. D., Arabi, Y., Shinagawa, N., et al. (1978). Identification of Clostridium difficile as a cause of pseudomembranous colitis. Br. Med. J. 1, 695. doi: 10.1136/bmj.1.6114.695

Griffiths, D., Fawley, W., Kachrimanidou, M., Bowden, R., Crook, D. W., Fung, R., et al. (2010). Multilocus sequence typing of Clostridium difficile. J. Clin. Microbiol. 48, 770-778. doi: 10.1128/JCM.01796-09

Hawkey, P. M., Marriott, C., Liu, W. E., Jian, Z. J., Gao, Q., Ling, T. K., et al. (2013). Molecular epidemiology of Clostridium difficile infection in a major Chinese hospital: an underrecognized problem in Asia? J. Clin. Microbiol. 51, 3308-3313. doi: 10.1128/JCM.00587-13

Huang, H., Weintraub, A., Fang, H., Wu, S., Zhang, Y., and Nord, C. E. (2010). Antimicrobial susceptibility and heteroresistance in Chinese Clostridium difficile strains. Anaerobe 16, 633-635. doi: 10.1016/j.anaerobe.2010. 09.002

Huang, H., Wu, S., Wang, M., Zhang, Y., Fang, H., Palmgren, A. C., et al. (2009). Clostridium difficile infections in a Shanghai hospital: antimicrobial resistance, toxin profiles and ribotypes. Int. J. Antimicrob. Agents 33, 339-342. doi: 10.1016/j.ijantimicag.2008.09.022

Indra, A., Huhulescu, S., Schneeweis, M., Hasenberger, P., Kernbichler, S., Fiedler, A., et al. (2008). Characterization of Clostridium difficile isolates using capillary gel electrophoresis-based PCR ribotyping. J. Med. Microbiol. 57, 13771382. doi: 10.1099/jmm.0.47714-0

Joost, I., Speck, K., Herrmann, M., and Von Muller, L. (2009). Characterisation of Clostridium difficile isolates by $\operatorname{spA}$ and tcdC gene sequencing. Int. J. Antimicrob. Agents 33(Suppl. 1), S13-S18. doi: 10.1016/S0924-8579(09)70010-X

Kato, H., Yokoyama, T., and Arakawa, Y. (2005). Typing by sequencing the slpA gene of Clostridium difficile strains causing multiple outbreaks in Japan. J. Med. Microbiol. 54, 167-171. doi: 10.1099/jmm.0.45807-0

Killgore, G., Thompson, A., Johnson, S., Brazier, J., Kuijper, E., Pepin, J., et al. (2008). Comparison of seven techniques for typing international epidemic strains of Clostfidium difficile: restriction endonuclease analysis, 
pulsed-field gel electrophoresis, PCR-ribotyping, multilocus sequence typing, multilocus variable-number tandem-repeat analysis, amplified fragment length polymorphism, and surface layer protein A gene sequence typing. J. Clin. Microbiol. 46, 431-437.

Kim, H., Riley, T. V., Kim, M., Kim, C. K., Yong, D., Lee, K., et al. (2008). Increasing prevalence of toxin A-negative, toxin B-positive isolates of Clostridium difficile in Korea: impact on laboratory diagnosis. J. Clin. Microbiol. 46, 1116-1117. doi: 10.1128/JCM.01188-07

King, A. M., Mackin, K. E., and Lyras, D. (2015). Emergence of toxin A-negative, toxin B-positive Clostridium difficile strains: epidemiological and clinical considerations. Future Microbiol. 10, 1-4. doi: 10.2217/fmb.14.115

Knight, D. R., Elliott, B., Chang, B. J., Perkins, T. T., and Riley, T. V. (2015). Diversity and evolution in the genome of Clostridium difficile. Clin. Microbiol. Rev. 28, 721-741. doi: 10.1128/CMR.00127-14

Marsh, J. W., O’leary, M. M., Shutt, K. A., Pasculle, A. W., Johnson, S., Gerding, D. N., et al. (2006). Multilocus variable-number tandem-repeat analysis for investigation of Clostridium difficile transmission in Hospitals. J. Clin. Microbiol. 44, 2558-2566. doi: 10.1128/JCM.02364-05

McDonald, L. C., Killgore, G. E., Thompson, A., Owens, R. C. Jr., Kazakova, S. V., Sambol, S. P., et al. (2005). An epidemic, toxin gene-variant strain of Clostridium difficile. N. Engl. J. Med. 353, 2433-2441. doi: 10.1056/NEJMoa051590

Popoff, M. R., Rubin, E. J., Gill, D. M., and Boquet, P. (1988). Actin-specific ADPribosyltransferase produced by a Clostridium difficile strain. Infect. Immun. 56, 2299-2306.

Rupnik, M., Wilcox, M. H., and Gerding, D. N. (2009). Clostridium difficile infection: new developments in epidemiology and pathogenesis. Nat. Rev. Microbiol. 7, 526-536. doi: 10.1038/nrmicro2164

Stewart, D. B., Berg, A., and Hegarty, J. (2013). Predicting recurrence of C. difficile colitis using bacterial virulence factors: binary toxin is the key. J. Gastrointest. Surg. 17, 118-124; discussion 124-115. doi: 10.1007/s11605-012-2056-6

Tian, T. T., Zhao, J. H., Yang, J., Qiang, C. X., Li, Z. R., Chen, J., et al. (2016). Molecular characterization of Clostridium difficile isolates from human subjects and the environment. PLoS ONE 11:e0151964. doi: 10.1371/journal.pone.0151964

van den Berg, R. J., Schaap, I., Templeton, K. E., Klaassen, C. H., and Kuijper, E. J. (2007). Typing and subtyping of Clostridium difficile isolates by using multiple-locus variable-number tandem-repeat analysis. J. Clin. Microbiol. 45, 1024-1028. doi: 10.1128/JCM.02023-06

Wang, X., Cai, L., Yu, R., Huang, W., and Zong, Z. (2014). ICU-Onset Clostridium difficile infection in a university hospital in China: a prospective cohort study. PLoS ONE 9:e111735. doi: 10.1371/journal.pone. 0111735

Yan, Q., Zhang, J., Chen, C., Zhou, H., Du, P., Cui, Z., et al. (2013). Multilocus sequence typing (MLST) analysis of 104 Clostridium difficile strains isolated from China. Epidemiol. Infect. 141, 195-199. doi: 10.1017/S0950268812 000453

Ye, G. Y., Li, N., Chen, Y. B., Lv, T., Shen, P., Gu, S. L., et al. (2015). Clostridium difficile carriage in healthy pregnant women in China. Anaerobe 37, 54-57. doi: 10.1016/j.anaerobe.2015.11.010

Zhou, F. F., Wu, S., Klena, J. D., and Huang, H. H. (2014). Clinical characteristics of Clostridium difficile infection in hospitalized patients with antibiotic-associated diarrhea in a university hospital in China. Eur. J. Clin. Microbiol. Infect. Dis. 33, 1773-1779. doi: 10.1007/s10096-014-2132-9

Conflict of Interest Statement: The authors declare that the research was conducted in the absence of any commercial or financial relationships that could be construed as a potential conflict of interest.

Copyright (C) 2016 Cheng, Xiao, Kudinha, Kong, Xu, Sun, Zhang, Fan, Xie and Xu. This is an open-access article distributed under the terms of the Creative Commons Attribution License (CC BY). The use, distribution or reproduction in other forums is permitted, provided the original author(s) or licensor are credited and that the original publication in this journal is cited, in accordance with accepted academic practice. No use, distribution or reproduction is permitted which does not comply with these terms. 